\title{
Measurement of deeply virtual Compton scattering at HERA
}

\section{Journal Article}

\section{Author(s):}

H1 Collaboration; Aktas, A.; Baumgartner, Simon; Berger, N.; Erdmann, Wolfram; Grab, Christoph; List, Benno; Mangano, Boris; Meer, David; Schöning, André; Weber, Ronald Mike; Zimmermann, T.; et al.

\section{Publication date:}

2005-09

\section{Permanent link:}

https://doi.org/10.3929/ethz-b-000031607

\section{Rights / license:}

Creative Commons Attribution 4.0 International

\section{Originally published in:}

The European Physical Journal C 44(1), https://doi.org/10.1140/epjc/s2005-02345-3 


\title{
Measurement of deeply virtual Compton scattering at HERA
}

\author{
The H1 Collaboration
}

A. Aktas $^{10}$, V. Andreev ${ }^{26}$, T. Anthonis ${ }^{4}$, S. Aplin ${ }^{10}$, A. Asmone ${ }^{34}$, A. Astvatsatourov ${ }^{4}$, A. Babaev ${ }^{25}$, S. Backovic ${ }^{31}$, J. Bähr ${ }^{39}$, A. Baghdasaryan ${ }^{38}$, P. Baranov ${ }^{26}$, E. Barrelet ${ }^{30}$, W. Bartel ${ }^{10}$, S. Baudrand ${ }^{28}$, S. Baumgartner ${ }^{40}$, J. Becker ${ }^{41}$, M. Beckingham ${ }^{10}$, O. Behnke ${ }^{13}$, O. Behrendt ${ }^{7}$, A. Belousov ${ }^{26}$, Ch. Berger ${ }^{1}$, N. Berger ${ }^{40}$, J.C. Bizot ${ }^{28}$, M.-O. Boenig ${ }^{7}$, V. Boudry ${ }^{29}$, J. Bracinik ${ }^{27}$, G. Brandt ${ }^{13}$, V. Brisson ${ }^{28}$, D.P. Brown ${ }^{10}$, D. Bruncko ${ }^{16}$, F.W. Büsser ${ }^{11}$, A. Bunyatyan ${ }^{12,38}$, G. Buschhorn ${ }^{27}$, L. Bystritskaya ${ }^{25}$, A.J. Campbell ${ }^{10}$, S. Caron ${ }^{1}$, F. Cassol-Brunner ${ }^{22}$, K. Cerny ${ }^{33}$, V. Cerny ${ }^{16,47}$, V. Chekelian ${ }^{27}$, J.G. Contreras ${ }^{23}$, J.A. Coughlan ${ }^{5}$, B.E. Cox ${ }^{21}$, G. Cozzika ${ }^{9}$, J. Cvach ${ }^{32}$, J.B. Dainton ${ }^{18}$, W.D. Dau ${ }^{15}$, K. Daum ${ }^{37,43}$, Y. de Boer ${ }^{25}$, B. Delcourt ${ }^{28}$, R. Demirchyan ${ }^{38}$, A. De Roeck ${ }^{10,45}$, K. Desch ${ }^{11}$, E.A. De Wolf ${ }^{4}$, C. Diaconu ${ }^{22}$, V. Dodonov ${ }^{12}$, A. Dubak ${ }^{31,46}$, G. Eckerlin ${ }^{10}$, V. Efremenko ${ }^{25}$, S. Egli ${ }^{36}$, R. Eichler ${ }^{36}$, F. Eisele ${ }^{13}$, M. Ellerbrock ${ }^{13}$, E. Elsen ${ }^{10}$, W. Erdmann ${ }^{40}$, S. $\operatorname{Essenov}^{25}$, A. Falkewicz ${ }^{6}$, P.J.W. Faulkner ${ }^{3}$, L. Favart ${ }^{4}$, A. Fedotov ${ }^{25}$, R. Felst ${ }^{10}$, J. Ferencei ${ }^{16}$, L. Finke ${ }^{11}$, M. Fleischer ${ }^{10}$, P. Fleischmann ${ }^{10}$, Y.H. Fleming ${ }^{10}$, G. Flucke ${ }^{10}$, A. Fomenko ${ }^{26}$, I. Foresti ${ }^{41}$, G. Franke ${ }^{10}$, T. Frisson ${ }^{29}$, E. Gabathuler ${ }^{18}$, E. Garutti ${ }^{10}$, J. Gayler ${ }^{10}$, C. Gerlich ${ }^{13}$, S. Ghazaryan ${ }^{38}$, S. Ginzburgskaya ${ }^{25}$, A. Glazov ${ }^{10}$, I. Glushkov ${ }^{39}$, L. Goerlich ${ }^{6}$, M. Goettlich ${ }^{10}$, N. Gogitidze ${ }^{26}$, S. Gorbounov ${ }^{39}$, C. Goyon ${ }^{22}$, C. Grab ${ }^{40}$, T. Greenshaw ${ }^{18}$, M. Gregori ${ }^{19}$, B.R. Grell ${ }^{10}$, G. Grindhammer ${ }^{27}$, C. Gwilliam ${ }^{21}$, D. Haidt ${ }^{10}$, L. Hajduk ${ }^{6}$, J. Haller ${ }^{13}$, M. Hansson ${ }^{20}$, G. Heinzelmann ${ }^{11}$, R.C.W. Henderson ${ }^{17}$, H. Henschel ${ }^{39}$, O. Henshaw ${ }^{3}$, G. Herrera ${ }^{24}$, M. Hildebrandt ${ }^{36}$, K.H. Hiller ${ }^{39}$, D. Hoffmann ${ }^{22}$, R. Horisberger ${ }^{36}$, A. Hovhannisyan ${ }^{38}$, M. Ibbotson ${ }^{21}$, M. Ismail ${ }^{21}$, M. Jacquet ${ }^{28}$, L. Janauschek ${ }^{27}$, X. Janssen ${ }^{10}$, V. Jemanov ${ }^{11}$, L. Jönsson ${ }^{20}$, D.P. Johnson ${ }^{4}$, H. Jung ${ }^{20,10}$, M. Kapichine ${ }^{8}$, J. Katzy ${ }^{10}$, N. Keller ${ }^{41}$, I.R. Kenyon ${ }^{3}$, C. Kiesling ${ }^{27}$, M. Klein ${ }^{39}$, C. Kleinwort ${ }^{10}$, T. Klimkovich ${ }^{10}$, T. Kluge ${ }^{10}$, G. Knies ${ }^{10}$, A. Knutsson ${ }^{20}$, V. Korbel ${ }^{10}$, P. Kostka ${ }^{39}$, R. Koutouev ${ }^{12}$, K. Krastev ${ }^{35}$, J. Kretzschmar ${ }^{39}$, A. Kropivnitskaya ${ }^{25}$, K. Krüger ${ }^{14}$, J. Kückens ${ }^{10}$, M.P.J. Landon ${ }^{19}$, W. Lange ${ }^{39}$, T. Laštovička ${ }^{39,33}$, G. Laštovička-Medin ${ }^{31}$, P. Laycock ${ }^{18}$, A. Lebedev ${ }^{26}$, B. Leißner ${ }^{1}$, V. Lendermann ${ }^{14}$, S. Levonian ${ }^{10}$, L. Lindfeld ${ }^{41}$, K. Lipka ${ }^{39}$, B. List ${ }^{40}$, E. Lobodzinska ${ }^{39,6}$, N. Loktionova ${ }^{26}$, R. Lopez-Fernandez ${ }^{10}$, V. Lubimov ${ }^{25}$, A.-I. Lucaci-Timoce ${ }^{10}$, H. Lueders ${ }^{11}$, D. Lüke ${ }^{7,10}$, T. Lux ${ }^{11}$, L. Lytkin ${ }^{12}$, A. Makankine ${ }^{8}$, N. Malden ${ }^{21}$, E. Malinovskii ${ }^{26}$, S. Mangano ${ }^{40}$, P. Marage ${ }^{4}$, R. Marshall ${ }^{21}$, M. Martisikova ${ }^{10}$, H.-U. Martyn ${ }^{1}$, S.J. Maxfield ${ }^{18}$, D. Meer ${ }^{40}$, A. Mehta ${ }^{18}$, K. Meier ${ }^{14}$, A.B. Meyer ${ }^{11}$, H. Meyer ${ }^{37}$, J. Meyer ${ }^{10}$, S. Mikocki ${ }^{6}$, I. Milcewicz-Mika ${ }^{6}$, D. Milstead ${ }^{18}$, D. Mladenov ${ }^{35}$, A. Mohamed ${ }^{18}$, F. Moreau ${ }^{29}$, A. Morozov ${ }^{8}$, J.V. Morris ${ }^{5}$, M.U. Mozer ${ }^{13}$, K. Müller ${ }^{41}$, P. Murín ${ }^{16,44}$, K. Nankov ${ }^{35}$, B. Naroska ${ }^{11}$, Th. Naumann ${ }^{39}$, P.R. Newman ${ }^{3}$, C. Niebuhr ${ }^{10}$, A. Nikiforov ${ }^{27}$, D. Nikitin ${ }^{8}$, G. Nowak ${ }^{6}$, M. Nozicka ${ }^{33}$, R. Oganezov ${ }^{38}$, B. Olivier ${ }^{3}$, J.E. Olsson ${ }^{10}$, S. Osman ${ }^{20}$, D. Ozerov 25 , V. Palichik ${ }^{8}$, I. Panagoulias ${ }^{10}$, T. Papadopoulou ${ }^{10}$, C. Pascaud ${ }^{28}$, G.D. Patel ${ }^{18}$, M. Peez ${ }^{29}$, E. Perez ${ }^{9}$, D. Perez-Astudillo ${ }^{23}$, A. Perieanu ${ }^{10}$, A. Petrukhin ${ }^{25}$, D. Pitzl ${ }^{10}$, R. Plačakytë ${ }^{27}$, B. Portheault ${ }^{28}$, B. Povh $^{12}$, P. Prideaux ${ }^{18}$, N. Raicevic $^{31}$, P. Reimer ${ }^{32}$, A. Rimmer ${ }^{18}$, C. Risler ${ }^{10}$, E. Rizvi ${ }^{19}$, P. Robmann ${ }^{41}$, B. Roland ${ }^{4}$, R. Roosen ${ }^{4}$, A. Rostovtsev ${ }^{25}$,

Z. Rurikova ${ }^{27}$, S. Rusakov ${ }^{26}$, F. Salvaire ${ }^{11}$, D.P.C. Sankey ${ }^{5}$, E. Sauvan ${ }^{22}$, S. Schätzel ${ }^{10}$, F.-P. Schilling ${ }^{10}$, S. Schmidt ${ }^{10}$, S. Schmitt ${ }^{41}$, C. Schmitz ${ }^{41}$, L. Schoeffel ${ }^{9}$, A. Schöning ${ }^{40}$, V. Schröder ${ }^{10}$, H.-C. Schultz-Coulon ${ }^{14}$, K. Sedlák ${ }^{32}$, F. Sefkow ${ }^{10}$, I. Sheviakov ${ }^{26}$, L.N. Shtarkov ${ }^{26}$, Y. Sirois ${ }^{29}$, T. Sloan ${ }^{17}$, P. Smirnov ${ }^{26}$, Y. Soloviev ${ }^{26}$, D. South ${ }^{10}$, V. Spaskov ${ }^{8}$, A. Specka ${ }^{29}$, B. Stella ${ }^{34}$, J. Stiewe ${ }^{14}$, I. Strauch ${ }^{10}$, U. Straumann ${ }^{41}$, V. Tchoulakov ${ }^{8}$, G. Thompson ${ }^{19}$,

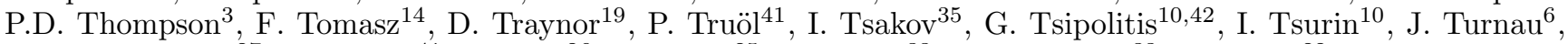
E. Tzamariudaki ${ }^{27}$, M. Urban ${ }^{41}$, A. Usik ${ }^{26}$, D. Utkin ${ }^{25}$, S. Valkár ${ }^{33}$, A. Valkárová ${ }^{33}$, C. Vallée 22 , P. Van Mechelen ${ }^{4}$, N. Van Remortel ${ }^{4}$, A. Vargas Trevino ${ }^{7}$, Y. Vazdik ${ }^{26}$, C. Veelken ${ }^{18}$, A. Vest ${ }^{1}$, S. Vinokurova ${ }^{10}$, V. Volchinski ${ }^{38}$, B. Vujicic ${ }^{27}$, K. Wacker ${ }^{7}$, J. Wagner ${ }^{10}$, G. Weber ${ }^{11}$, R. Weber ${ }^{40}$, D. Wegener ${ }^{7}$, C. Werner ${ }^{13}$, N. Werner ${ }^{41}$, M. Wessels $^{10}$, B. Wessling ${ }^{10}$, C. Wigmore ${ }^{3}$, Ch. Wissing ${ }^{7}$, R. Wolf ${ }^{13}$, E. Wünsch ${ }^{10}$, S. Xella ${ }^{41}$, W. Yan ${ }^{10}$, V. Yeganov ${ }^{38}$, J. Žáček $^{33}$, J. Zálešák ${ }^{32}$, Z. Zhang ${ }^{28}$, A. Zhelezov ${ }^{25}$, A. Zhokin ${ }^{25}$, J. Zimmermann ${ }^{27}$, T. Zimmermann ${ }^{40}$, H. Zohrabyan ${ }^{38}$,

F. Zomer ${ }^{28}$

1 I. Physikalisches Institut der RWTH, Aachen, Germany ${ }^{\mathrm{a}}$

2 III. Physikalisches Institut der RWTH, Aachen, Germany ${ }^{\mathrm{a}}$

3 School of Physics and Astronomy, University of Birmingham, Birmingham, $\mathrm{UK}^{\mathrm{b}}$

${ }^{4}$ Inter-University Institute for High Energies ULB-VUB, Brussels; Universiteit Antwerpen, Antwerpen; Belgium ${ }^{\mathrm{c}}$

5 Rutherford Appleton Laboratory, Chilton, Didcot, UK ${ }^{\mathrm{b}}$

6 Institute for Nuclear Physics, Cracow, Poland ${ }^{\mathrm{d}}$

7 Institut für Physik, Universität Dortmund, Dortmund, Germany ${ }^{a}$

8 Joint Institute for Nuclear Research, Dubna, Russia 
9 CEA, DSM/DAPNIA, CE-Saclay, Gif-sur-Yvette, France

10 DESY, Hamburg, Germany

11 Institut für Experimentalphysik, Universität Hamburg, Hamburg, Germany ${ }^{a}$

12 Max-Planck-Institut für Kernphysik, Heidelberg, Germany

13 Physikalisches Institut, Universität Heidelberg, Heidelberg, Germany ${ }^{a}$

14 Kirchhoff-Institut für Physik, Universität Heidelberg, Heidelberg, Germany ${ }^{a}$

15 Institut für experimentelle und angewandte Physik, Universität Kiel, Kiel, Germany

16 Institute of Experimental Physics, Slovak Academy of Sciences, Košice, Slovak Republic ${ }^{f}$

17 Department of Physics, University of Lancaster, Lancaster, UK ${ }^{\mathrm{b}}$

18 Department of Physics, University of Liverpool, Liverpool, $\mathrm{UK}^{\mathrm{b}}$

19 Queen Mary and Westfield College, London, UK ${ }^{\mathrm{b}}$

20 Physics Department, University of Lund, Lund, Sweden ${ }^{\mathrm{g}}$

21 Physics Department, University of Manchester, Manchester, $\mathrm{UK}^{\mathrm{b}}$

22 CPPM, CNRS/IN2P3 - Univ Mediterranee, Marseille - France

23 Departamento de Fisica Aplicada, CINVESTAV, Mérida, Yucatán, México ${ }^{k}$

24 Departamento de Fisica, CINVESTAV, Méxicok $^{k}$

${ }^{25}$ Institute for Theoretical and Experimental Physics, Moscow, Russia ${ }^{1}$

26 Lebedev Physical Institute, Moscow, Russia ${ }^{\mathrm{e}}$

27 Max-Planck-Institut für Physik, München, Germany

28 LAL, Université de Paris-Sud, IN2P3-CNRS, Orsay, France

29 LLR, Ecole Polytechnique, IN2P3-CNRS, Palaiseau, France

30 LPNHE, Universités Paris VI and VII, IN2P3-CNRS, Paris, France

31 Faculty of Science, University of Montenegro, Podgorica, Serbia and Montenegro

32 Institute of Physics, Academy of Sciences of the Czech Republic, Praha, Czech Republic ${ }^{\mathrm{e}, \mathrm{i}}$

33 Faculty of Mathematics and Physics, Charles University, Praha, Czech Republic ${ }^{\mathrm{e}, \mathrm{i}}$

34 Dipartimento di Fisica Università di Roma Tre and INFN Roma 3, Roma, Italy

35 Institute for Nuclear Research and Nuclear Energy, Sofia, Bulgaria

36 Paul Scherrer Institut, Villingen, Switzerland

37 Fachbereich C, Universität Wuppertal, Wuppertal, Germany

38 Yerevan Physics Institute, Yerevan, Armenia

39 DESY, Zeuthen, Germany

40 Institut für Teilchenphysik, ETH, Zürich, Switzerland ${ }^{\mathrm{j}}$

41 Physik-Institut der Universität Zürich, Zürich, Switzerland ${ }^{\mathrm{j}}$

42 Also at Physics Department, National Technical University, Zografou Campus, GR-15773 Athens, Greece

43 Also at Rechenzentrum, Universität Wuppertal, Wuppertal, Germany

44 Also at University of P.J. Šafárik, Košice, Slovak Republic

45 Also at CERN, Geneva, Switzerland

46 Also at Max-Planck-Institut für Physik, München, Germany

47 Also at Comenius University, Bratislava, Slovak Republic

Received: 19 May 2005 / Revised version: 23 June 2005 /

Published online: 19 August 2005 - (C) Springer-Verlag / Società Italiana di Fisica 2005

Abstract. A measurement is presented of elastic deeply virtual Compton scattering $\gamma^{*} p \rightarrow \gamma p$ made using $e^{+} p$ collision data corresponding to a luminosity of $46.5 \mathrm{pb}^{-1}$, taken with the $\mathrm{H} 1$ detector at HERA. The cross section is measured as a function of the photon virtuality, $Q^{2}$, the invariant mass of the $\gamma^{*} \mathrm{p}$ system, $W$, and for the first time, differentially in the squared momentum transfer at the proton vertex, $t$, in the kinematic range $2<Q^{2}<80 \mathrm{GeV}^{2}, 30<W<140 \mathrm{GeV}$ and $|t|<1 \mathrm{GeV}^{2}$. QCD based calculations at next-to-leading order using generalized parton distributions can describe the data, as can colour dipole model predictions.

a Supported by the Bundesministerium für Bildung und Forschung, FRG, under contract numbers 05 H1 1GUA /1, $05 \mathrm{H} 1$ 1PAA /1, $05 \mathrm{H} 1$ 1PAB /9, $05 \mathrm{H} 1$ 1PEA /6, $05 \mathrm{H} 1$ $1 \mathrm{VHA} / 7$ and $05 \mathrm{H} 11 \mathrm{VHB} / 5$

b Supported by the UK Particle Physics and Astronomy Research Council, and formerly by the UK Science and Engineering Research Council

c Supported by FNRS-FWO-Vlaanderen, IISN-IIKW and IWT and by Interuniversity Attraction Poles Programme, Bel-

\footnotetext{
gian Science Policy

d Partially Supported by the Polish State Committee for Scientific Research, SPUB/DESY/P003/DZ 118/2003/2005

e Supported by the Deutsche Forschungsgemeinschaft

f Supported by VEGA SR grant no. 2/4067/ 24

g Supported by the Swedish Natural Science Research Council

i Supported by the Ministry of Education of the Czech Republic under the projects INGO-LA116/2000 and LN00A006,
} 


\section{Introduction}

Measurements of the deep-inelastic scattering (DIS) of leptons and nucleons allow the extraction of Parton Distribution Functions (PDFs) which describe the longitudinal momentum carried by the quarks, anti-quarks and gluons that make up the fast-moving nucleons. While these PDFs provide crucial input to perturbative Quantum Chromodynamic (QCD) calculations of processes involving hadrons, they do not provide a complete picture of the partonic structure of nucleons. In particular, PDFs contain neither information on the correlations between partons nor on their transverse motion. This missing information can be provided by measurements of processes in which the nucleon remains intact, such as the exclusive production of light meson states in lepton-nucleon collisions, and is encoded in Generalised Parton Distributions (GPDs) [1-4].

The simplest process sensitive to GPDs is deeply virtual Compton scattering (DVCS) (Fig. 1a), which is the diffractive scattering of a virtual photon off a proton [5-10], $\gamma^{*} p \rightarrow \gamma p$. In the present analysis DVCS is accessed through the reaction:

$$
e^{+} p \rightarrow e^{+} \gamma p
$$

This process is of particular interest as it has both a clear experimental signature and is calculable in perturbative QCD: it does not suffer from the uncertainties caused by the lack of understanding of the meson wave function that plague exclusive vector meson electroproduction ${ }^{1}$.

The reaction studied receives contributions from both the DVCS process, whose origin lies in the strong interaction, and the purely electromagnetic Bethe-Heitler (BH) process (Figs. 1b and 1c), where the photon is emitted from the positron. The $\mathrm{BH}$ cross section can be precisely calculated in QED using elastic proton form factors. Here, the DVCS cross section is obtained by subtracting the BH contribution from the total cross section, which is possible since the interference contribution vanishes [10], as this measurement is integrated over azimuthal angles.

The first measurements of the DVCS cross section at high energy were obtained by H1 [11] and ZEUS [12] and the

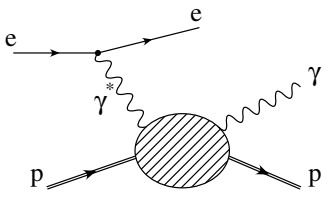

(a)

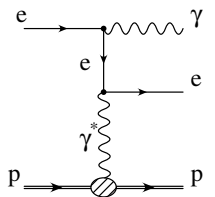

(b)

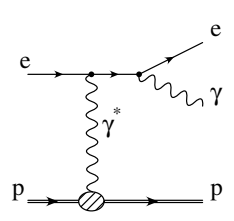

(c)
Fig. 1. Diagrams illustrating the DVCS a and the Bethe-Heitler $\mathbf{b}$ and $\mathbf{c}$ processes

by GAUK grant no $175 / 2000$

j Supported by the Swiss National Science Foundation

k Supported by CONACYT, México, grant 400073-F

1 Partially Supported by Russian Foundation for Basic Research, grant no. 00-15-96584

1 The purely perturbative approach adopted here is expected to break down in the absence of a hard scale, in which case it might be necessary to take into account the hadronic structure of the real photon in the calculations. helicity asymmetry in DVCS has been measured at lower energy with polarised lepton beams by HERMES [13] and CLAS [14].

In this paper, a measurement of the DVCS cross section is presented, based on data collected with the $\mathrm{H} 1$ detector at HERA in the years 1996 to 2000. These data correspond to a luminosity of $46.5 \mathrm{pb}^{-1}$, a factor of 4 larger than the luminosity used in the previous H1 publication [11], which is based only on 1997 data. The cross section is presented as a function of the photon virtuality, $Q^{2}$, the invariant mass of the $\gamma^{*}$ p system, $W$, and the squared momentum transfer at the proton vertex, $t$.

\section{Generalized parton distributions and theoretical predictions}

The leading order diagram for DVCS in positron proton scattering is shown in Fig. 2a and a diagram that contributes at next-to-leading order in Fig. 2b. The transition from a virtual photon to a real photon forces the fractional momenta of the two partons involved to be different ("skewed"). Hence, DVCS is sensitive to the correlations between partons in the proton which are encoded in the GPDs. In the presence of a hard scale, here $Q^{2}$, the DVCS scattering amplitude factorises $[3,6,7]$ into a hard part, calculable order by order in perturbative QCD, and the GPDs which contain the non-perturbative effects due to the structure of the proton.

\subsection{Generalized parton distributions}

The GPDs generalize and interpolate between the PDFs and elastic form factors. The PDFs contain information on the longitudinal momenta of the partons while form factors contain information on their transverse momenta, often in the form of sum rules related to charges, local currents and the energy-momentum tensor of QCD. GPDs have simple physical significance in light-cone coordinates (or the infinite momentum frame), where they represent the interference of two different wave functions, one of a parton having a momentum fraction $x+\xi$ and the other of a parton with a momentum fraction $x-\xi$, as is illustrated in Fig. 2. Besides the longitudinal momentum fraction variables $\xi$ (called skewedness) and $x$, GPDs depend on $t$, the square of the four-momentum exchanged at the hadron vertex. GPDs

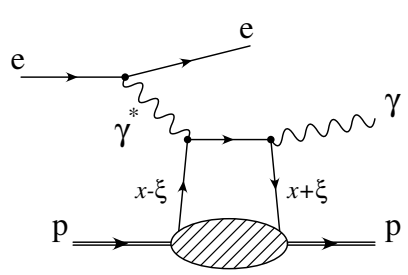

(a)

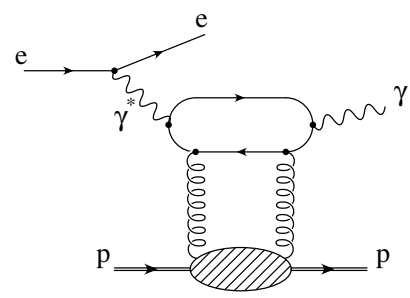

(b)
Fig. 2. Examples of diagrams for the DVCS process a at leading order, $\mathbf{b}$ at next-to-leading order 
are defined at a starting scale $\mu^{2}$ and their $Q^{2}$ evolution is generated by perturbative QCD.

There are two different types of GPDs (for a quark $q$ or a gluon $g)$ in the unpolarised case $^{2}: H^{q, g}(x, \xi, t)$ and $E^{q, g}(x, \xi, t)$. While the $E^{q, g}$ distributions have no equivalent in the ordinary PDF approach, the $H^{q, g}$ reduce to the usual PDFs in the forward limit $(\xi=0, t=0)$, i.e. $H^{q}(x, 0,0)=q(x)$ and $H^{g}(x, 0,0)=x g(x)$, where $q(x)$ and $g(x)$ are the ordinary parton distributions. The variable $x$ is defined in the range $[-1,1]$, with negative values corresponding to anti-quark distributions: $H^{q}(-x, 0,0)=$ $-\bar{q}(x)$. The gluon GPD is symmetric in $x$ in the forward limit: $H^{g}(-x, 0,0)=H^{g}(x, 0,0)$. The skewedness variable $\xi$ is related to the well known Bjorken- $x$ variable, $x_{B j}$, by $\xi=\alpha x_{B j} /\left(2-\alpha x_{B j}\right)$, where $\alpha=1+q^{\prime 2} / Q^{2}$ and $q^{\prime}$ denotes the four-momentum of the outgoing photon ${ }^{3}$. The first moments of the GPDs in $x$ are given by form factors [4].

Two different kinematic regions exist for GPDs with respect to the variables $x$ and $\xi$. The DGLAP region, where $|x|>\xi$ [16-19], corresponds to the emission and reabsorption of a quark, anti-quark or a gluon. The ERBL [20, $21]$ region, where $|x|<\xi$, corresponds to meson or gluon pair exchange. Each region has its own evolution equations.

The recent strong interest in GPDs was stimulated by the information they contain on the spin structure of the nucleon. In particular, GPDs are so far the only known means of probing the orbital motion of partons in the nucleon through Ji's Sum Rule [22], which relates unpolarised GPDs to the total angular momentum of the proton. DVCS measurements at HERA can provide constraints on this sum rule through their sensitivity to the GPDs.

\subsection{Theoretical predictions}

The measurements presented here are compared with NLO QCD calculations and predictions made using colour dipole approaches. In NLO QCD, the DVCS cross section has been calculated $[23,24]$ using two different GPD parameterisations [25]. The $t$ dependence of the GPDs is taken to be $e^{-b|t|}$. The MRST2001 [26] and CTEQ6 [27] parameterisations of the PDFs are used in the DGLAP region $(|x|>\xi)$. Thus $H$, which provides the main contribution to DVCS at small $x_{B j}$, is given at the starting scale $\mu$ by $H^{q}(x, \xi, t)=q(x) e^{-b|t|}$ for the quarks and $H^{g}(x, \xi, t)=x g(x) e^{-b|t|}$ for the gluons ${ }^{4}$. Both the skewing and the $Q^{2}$ dependence are generated dynamically. In the ERBL region $(|x|<\xi)$, these parameterisations have to be modified, ensuring a smooth continuation to the DGLAP region (for details see [25]). These GPD models are found to describe both the shape of the previous H1 DVCS cross

\footnotetext{
2 The notations used here are those introduced by Ji [15]; other notations can be found in the literature.

3 For the DVCS process, the outgoing photon is real $\left(q^{\prime 2}=0\right)$ and $\xi$ reduces to $x_{B j} /\left(2-x_{B j}\right)$. The forward limit corresponds to the case of inclusive DIS, where $q^{\prime 2}=-Q^{2}$ and thus $\xi=0$.

4 A different ansatz for GPDs has been used in [28] in a LO calculation of the DVCS cross section.
}

section measurements [11] and the single spin asymmetry measured by HERMES [13].

The DVCS cross section has also been calculated in the colour dipole approach, which is successful in describing both inclusive and diffractive scattering in the DIS regime at high energy. These predictions are based on a factorisation of the DVCS amplitude into the wave function for the photon to fluctuate into a $q \bar{q}$ pair, the cross section for this pair to interact with the proton and the outgoing photon wave function. If $s$-channel helicity is conserved in DVCS, the virtual photon must be transversely polarised. As the wave function of the transversely polarised $\gamma^{*}$ can select large dipole sizes, whose interactions are predominantly soft, DVCS constitutes a good probe of the transition between the perturbative and non-perturbative regimes of QCD. The various calculations differ in the way the dipole cross section is parameterised. Donnachie and Dosch [29] use soft and hard pomeron exchange depending on the size of the dipole. All parameters are determined from $p p$ and $\gamma^{*} p$ total cross section measurements. Favart and Machado [30] apply the saturation model of Golec-Biernat et al. [31] to the DVCS process and use DGLAP evolution [32], following the approach of Bartels, Golec-Biernat and Kowalski (BGBK) [33]. In both cases an exponential $t$-dependence, $e^{-b|t|}$, is assumed.

\section{Experimental procedure}

\section{$3.1 \mathrm{H} 1$ detector}

A detailed description of the $\mathrm{H} 1$ detector can be found in [34]. Here only the detector components relevant for the present analysis are described. The SpaCal [35], a lead scintillating fibre calorimeter, covers the backward ${ }^{5}$ region of the $\mathrm{H} 1$ detector $\left(153^{\circ}<\theta<177.5^{\circ}\right)$. Its energy resolution for electromagnetic showers is $\sigma(E) / E \simeq$ $7.1 \% / \sqrt{E / \mathrm{GeV}} \oplus 1 \%$. The liquid argon (LAr) calorimeter $\left(4^{\circ} \leq \theta \leq 154^{\circ}\right)$ is situated inside a solenoidal magnet. The energy resolution for electromagnetic showers is $\sigma(E) / E \simeq 11 \% / \sqrt{E / \mathrm{GeV}}$ as obtained from test beam measurements [36]. The backward drift chamber (BDC), placed in front of the SpaCal, measures track segments for charged particles entering the SpaCal from the interaction region. These are used to identify the scattered positron and to determine its position with a resolution of $0.5 \mathrm{~mm}$ in the radial and $2.5 \mathrm{~mm}$ in the azimuthal direction. The main component of the central tracking detector is the central jet chamber (CJC) which consists of two $2 \mathrm{~m}$ long coaxial cylindrical drift chambers, with wires parallel to the beam direction. The measurement of charged particle transverse momenta is performed in a magnetic field of $1.15 \mathrm{~T}$, uniform over the full tracker volume. The forward components of the detector, used here to tag hadronic activity at large

\footnotetext{
${ }^{5} \mathrm{H} 1$ uses a right-handed coordinate system with $z$ axis along the beam direction, the $+z$ or "forward" direction being that of the outgoing proton beam. The polar angle $\theta$ is defined with respect to the $z$ axis and the pseudo-rapidity is given by $\eta=-\ln \tan \theta / 2$.
} 
pseudo-rapidity $(5 \lesssim \eta \lesssim 7)$, are the forward muon detector (FMD) and the proton remnant tagger (PRT). The FMD, designed to identify muons emitted in the forward direction, contains six planes of drift cells. It is used here to detect the particles produced when a proton dissociates and secondary interactions occur in the beampipe and adjacent material. Secondary particles, or the scattered proton, can also be detected by the PRT, which is located at $24 \mathrm{~m}$ from the interaction point and consists of layers of scintillator surrounding the beam pipe. The luminosity is determined from the rate of $\mathrm{BH}$ events measured in a luminosity monitor.

\subsection{Kinematics}

For DVCS, the final state photon does not originate from the positron and therefore the ratio of the DVCS to the $\mathrm{BH}$ cross sections is expected to increase when the photon is scattered in the forward direction. The analysis sample is thus selected by requiring a photon candidate in the LAr calorimeter and a positron candidate in the SpaCal calorimeter.

The reconstruction of the kinematic variables $Q^{2}, x_{B j}$ and $W$ relies on the polar angle measurements of the final state positron, $\theta_{e}$, and photon, $\theta_{\gamma}$ :

$$
\begin{aligned}
Q^{2} & =4 E_{0}^{2} \frac{\sin \theta_{\gamma}\left(1+\cos \theta_{e}\right)}{\sin \theta_{\gamma}+\sin \theta_{e}-\sin \left(\theta_{e}+\theta_{\gamma}\right)}, \\
x_{B j} & =\frac{E_{0}}{E_{p}} \frac{\sin \theta_{\gamma}+\sin \theta_{e}+\sin \left(\theta_{e}+\theta_{\gamma}\right)}{\sin \theta_{\gamma}+\sin \theta_{e}-\sin \left(\theta_{e}+\theta_{\gamma}\right)} \\
W^{2} & =\frac{Q^{2}}{x_{B j}}\left(1-x_{B j}\right),
\end{aligned}
$$

where $E_{0}$ and $E_{p}$ are the positron and proton beam energies, respectively. For the majority of the events, the scattered positron trajectory is not measured in the CJC and the event vertex cannot be determined. The polar angles of the positron and photon are then determined assuming that they come from the nominal event vertex. The square of the four-momentum transfer to the proton, $t$, is very well approximated by the square of the vector sum of the transverse momenta of the final state photon, $\boldsymbol{p}_{t_{\gamma}}$, and of the scattered positron, $\boldsymbol{p}_{t_{e}}$ :

$$
t \simeq-\left(\boldsymbol{p}_{t_{\gamma}}+\boldsymbol{p}_{t_{e}}\right)^{2}
$$

\subsection{Monte Carlo simulation}

Monte Carlo (MC) simulations are used to estimate the corrections that must be applied to the data due to the finite acceptance and resolution of the detector. Elastic DVCS events in $e p$ collisions are generated using the Monte Carlo generator MILOU [37], which is based on a NLO QCD cross section calculation [23, 24,38] (see Sect.2.2), and using a slope in $t$ of $b=6 \mathrm{GeV}^{-2}$. Higher order photon radiation from the incoming positron is implemented in the collinear approximation. DVCS events in which the proton dissociates into a baryonic system $Y$ are also simulated with the program MILOU using a $t$ slope of $b_{p d i s s}=1.5 \mathrm{GeV}^{-2}[39]$. The Monte Carlo generator COMPTON $2.1[40,41]$ is used to simulate both elastic and inelastic BH events. Hadronisation processes in inelastic $\mathrm{BH}$ events are simulated using the SOPHIA model [42]. Diffractive $\omega$ and $\phi$ meson events are generated with the DIFFVM Monte Carlo program [43]. The events generated using all these programs are passed through a detailed simulation of the $\mathrm{H} 1$ detector and are subject to the same reconstruction and analysis chain as the data.

\subsection{Event selection}

The data were obtained with the H1 detector when the HERA collider was operated with $820 \mathrm{GeV}$ (1996-1997) and $920 \mathrm{GeV}(1999-2000)$ protons and $27.6 \mathrm{GeV}$ positron beams. The data sample corresponds to an integrated luminosity of $46.5 \mathrm{pb}^{-1}, 11.5 \mathrm{pb}^{-1}$ of which were accumulated in 1996-1997 and $35 \mathrm{pb}^{-1}$ in 1999-2000. The event trigger used is based on the detection of an energy deposition greater than $6 \mathrm{GeV}$ in the electromagnetic section of the SpaCal calorimeter. Due to the different trigger settings, selected events in the 1996-1997 period are in the kinematic range $Q^{2}>2 \mathrm{GeV}^{2}$ while those in the 1999-2000 period are in the range $Q^{2}>4 \mathrm{GeV}^{2}$.

The DVCS event selection requires that the following criteria be fulfilled. The scattered positron must be detected in the SpaCal, have an energy larger than $15 \mathrm{GeV}$ and be validated by a track segment in the BDC. The photon must be measured in the LAr calorimeter with a transverse momentum $p_{t}>1 \mathrm{GeV}(1996-1997)$ or $p_{t}>1.5 \mathrm{GeV}$ (1999$2000)$ and a polar angle between $25^{\circ}$ and $145^{\circ}$. The scattered proton escapes undetected through the beam pipe. Events with more than one central track are rejected while events with one central track are only kept if that track is associated with the scattered positron. In order to reject inelastic and proton dissociation events, no further energy deposition in the LAr calorimeter with energy above $0.5 \mathrm{GeV}$ is allowed and no activity above the noise level is allowed in the PRT and FMD. The influence of QED radiative corrections is reduced by the requirement that the longitudinal momentum balance $\sum\left(E-P_{z}\right)>45 \mathrm{GeV}$. Here, $E$ denotes the energy and $P_{z}$ the momentum along the beam axis of the final state particles and the sum runs over all such particles. To enhance the DVCS signal with respect to the $\mathrm{BH}$ contribution and to ensure a large acceptance, the kinematic domain is explicitly restricted to $Q^{2}<80 \mathrm{GeV}^{2},|t|<1 \mathrm{GeV}^{2}$ and $30<W<140 \mathrm{GeV}$.

The selected sample contains 1243 events and is dominated by the DVCS contribution, but also contains contributions from the elastic $\mathrm{BH}$ process and from the (inelastic) BH and DVCS processes with proton dissociation, $e^{+} p \rightarrow e^{+} \gamma Y$, where the baryonic system $Y$ of mass $M_{Y}$ is not detected in the forward detectors.

As in previous H1 DVCS analyses [11,44], a control sample of $\mathrm{BH}$ events is also selected. Here, it is required that the positron be detected in the LAr and the photon 
in the SpaCal. It has been verified that the COMPTON $\mathrm{MC}$ correctly describes the normalisation and the shapes of the distributions of the kinematic variables for these events within an uncertainty of $5 \%$. Using events with a signal in the forward detectors, and subtracting the inelastic $\mathrm{BH}$ contribution, obtained from the COMPTON MC, the contribution of proton dissociation to the DVCS event sample is estimated to be $16 \pm 8 \%$ for the 1996-1997 data (lower $Q^{2}$ ) and $10 \pm 5 \%$ for the 1999-2000 data. The other backgrounds considered are diffractive $\omega$ and $\phi$ production, with decay modes to final states including photons. The main backgrounds originate from the decays $\omega \rightarrow \pi^{0} \gamma$ and $\phi \rightarrow K_{L}^{0} K_{S}^{0}$ followed by the decay $K_{S}^{0} \rightarrow \pi^{0} \pi^{0}$. The contribution of these processes to the DVCS sample is estimated to be below $3.5 \%$ for the data taken in 1996-1997 and below $1 \%$ for that taken in 1999-2000.

In Fig. 3 the data are compared with the sum of the $\mathrm{MC}$ expectations. The $\mathrm{BH}$ contributions and the $\omega$ and $\phi$ backgrounds are normalised to the luminosity. The DVCS contribution is normalised such that the sum of the DVCS, $\mathrm{BH}$ and diffractive vector meson contributions is equal to the total number of events in the data. The distributions of the energy and polar angle of the positron and the photon are shown in Figs. 3a-d. The coplanarity, shown in Fig. 3e, is defined to be the difference of the azimuthal angles of the electron and photon directions. It is related to the $p_{t^{-}}$ balance of the positron-photon system. The distribution of the invariant mass of the positron and the photon is presented in Fig. 3f. The sum of the MC contributions gives a good description of the shapes of the data distributions.

\subsection{Cross section measurement method}

To extract the cross section, the selected data are corrected for detector efficiencies, acceptance, bin-to-bin migrations and for initial state radiation from the positron using the Monte Carlo simulation. The inelastic BH contribution is subtracted bin by bin using the COMPTON Monte Carlo program. The contribution of DVCS events with proton dissociation is subtracted bin by bin using the MILOU Monte Carlo simulation. A 5\% correction is applied to correct for the loss of elastic DVCS events due to the requirement that there be no signal in the forward detectors. The background contributions from diffractive $\omega$ and $\phi$ production are also subtracted using the MC simulations.

In the leading twist approximation, the main contribution resulting from the interference of the BH and DVCS processes is proportional to the cosine of the azimuthal angle of the photon ${ }^{6}$. Since the present measurement is integrated over this angle, the overall contribution of the interference term is negligible. The elastic $\mathrm{BH}$ cross section can therefore be subtracted from the total $e^{+} p \rightarrow e^{+} \gamma p$ cross section in order to obtain the contribution from DVCS pro-

6 The azimuthal angle of the photon is defined as the angle between the plane formed by the incoming and scattered positron and that formed by the $\gamma^{*}$ and the scattered proton.
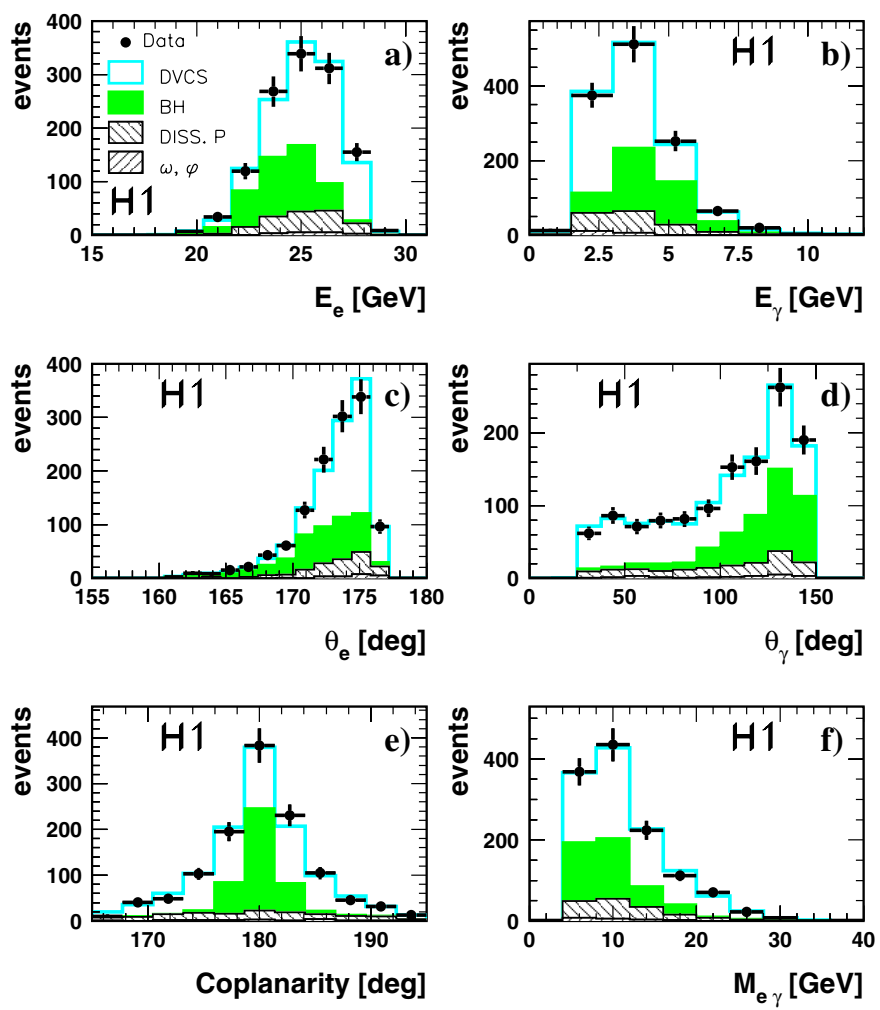

Fig. 3. Distributions of the energy of the scattered positron $\mathbf{a}$, the energy of the photon $\mathbf{b}$, the polar angle of the scattered positron $\mathbf{c}$, the polar angle of the photon $\mathbf{d}$, the coplanarity $\mathbf{e}$, and the positron-photon invariant mass $\mathbf{f}$. The data are compared with MC expectations for elastic DVCS, elastic BH, BH and DVCS with proton dissociation, and $\omega$ and $\phi$ diffractive backgrounds. The DVCS contribution is normalised such that the sum of the DVCS, BH and diffractive vector meson contributions is equal to the total number of events in the data. The normalisation of the other contributions is described in the text

cesses. This contribution is then converted to the $\gamma^{*} p \rightarrow \gamma p$ cross section using the equivalent photon approximation ${ }^{7}$ :

$$
\begin{aligned}
& \frac{\mathrm{d}^{3} \sigma[e p \rightarrow e \gamma p]}{\mathrm{d} y \mathrm{~d} Q^{2} \mathrm{~d} t}\left(Q^{2}, y, t\right) \\
& =\Gamma\left(Q^{2}, y\right) \frac{\mathrm{d} \sigma\left[\gamma^{*} p \rightarrow \gamma p\right]}{\mathrm{d} t}\left(Q^{2}, y, t\right),
\end{aligned}
$$

where the transverse photon flux $\Gamma$ is given by [45],

$$
\Gamma=\frac{\alpha\left(1-y+\frac{y^{2}}{2}\right)}{\pi y Q^{2}} \quad \text { with } \quad y=\frac{W^{2}+Q^{2}}{s} .
$$

Here, $s$ is the square of the $e p$ centre-of-mass energy.

The $t$ dependence is factorised according to:

$$
\frac{\mathrm{d} \sigma\left[\gamma^{*} p \rightarrow \gamma p\right]}{\mathrm{d} t}\left(Q^{2}, y, t\right)=\left.\frac{\mathrm{d} \sigma\left[\gamma^{*} p \rightarrow \gamma p\right]}{\mathrm{d} t}\right|_{t=0} e^{-b|t|}
$$

\footnotetext{
7 After integrating over azimuthal angles only transversely polarised $\gamma^{*}$ contribute to the DVCS process.
} 
The cross section $\sigma\left[\gamma^{*} p \rightarrow \gamma p\right]$ is extracted from equations (6) and (8) using an iterative procedure and fitting the $t$ integrated cross section with the form:

$$
\sigma\left[\gamma^{*} p \rightarrow \gamma p\right]\left(Q^{2}, y\right)=N \cdot y^{\delta / 2} \cdot\left(\frac{1}{Q^{2}}\right)^{n},
$$

where $\delta, n$ and $b$ are free parameters and $N$ is fixed by the integration of equation (6). More details can be found in [44].

The same method is used to extract $\sigma\left[\gamma^{*} p \rightarrow \gamma p\right]$ as a function of $Q^{2}$ and of $x_{B j}$.

\subsection{Systematic errors}

The main sources of systematic errors and their resulting uncertainty on the DVCS cross section measurements are:

- the subtraction of the DVCS proton dissociation background (typically $11 \%$ in $1996-1997,8 \%$ in $1999-2000$ and up to $20 \%$ in the highest $|t|$ bin) estimated using MC simulations with $b_{\text {pdiss }}=1.5 \pm 0.5 \mathrm{GeV}^{-2}$ and an $M_{Y}$ dependence $d \sigma / d M_{Y}^{2} \sim\left(1 / M_{Y}\right)^{2.0 \pm 0.3}$;

- the uncertainty on the acceptance correction factors (typically $10 \%$ and up to $25 \%$ in the highest $|t|$ bin) calculated by varying $b$ between 4 and $7 \mathrm{GeV}^{-2}$;

- the uncertainty on the determination of $\delta$ and $n$ used for the bin centre corrections (which ranges between 9 and $16 \%$ );

- the uncertainty on the BH subtraction (up to $7 \%$ for the highest $W$ bin);

- the uncertainties on the vertex position and the measurement of the scattered positron/photon angles (each contribution leading to up to $12 \%$ in the highest $|t|$ bin);

- the uncertainties on the positron/photon energies (each contribution leading to up to $12 \%$ in the highest $|t|$ bin);

- the noise in the CJC (typically 4\%), and in the FMD (up to $2 \%$ );

- the luminosity measurement (typically $2.5 \%$ ).

The total systematic error is found to be typically $25 \%$.

\section{Results}

\subsection{Cross sections}

The cross sections are determined separately for the two data taking periods, which cover different ranges in $Q^{2}$, and

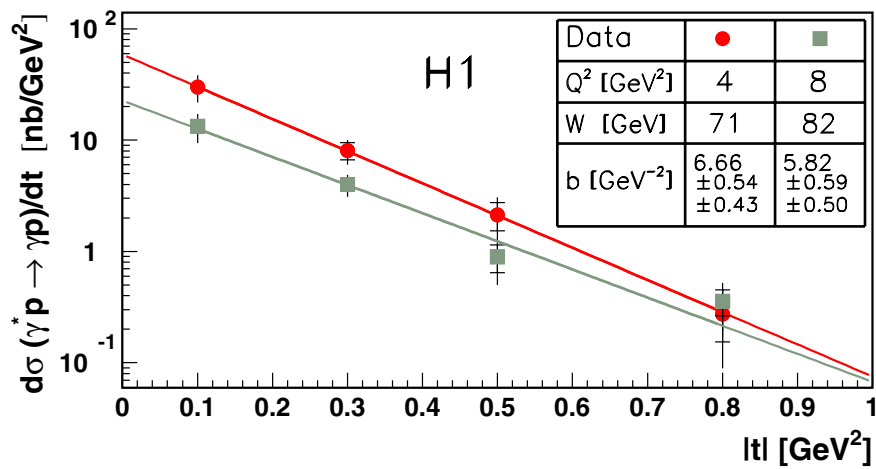

Fig. 4. The cross section $\gamma^{*} p \rightarrow \gamma p$ differential in $t$, for $Q^{2}=$ $4 \mathrm{GeV}^{2}$ at $W=71 \mathrm{GeV}$ and $Q^{2}=8 \mathrm{GeV}^{2}$ at $W=82 \mathrm{GeV}$. The inner error bars represent the statistical and the full error bars the quadratic sum of the statistical and systematic uncertainties. The lines represent the results of fits to the exponential form $e^{-b|t|}$, giving the values of $b$ shown in the insert (see Table 4)

are then combined. The $1996-1997$ period covers the kinematic range $2<Q^{2}<20 \mathrm{GeV}^{2}$ and $30<W<120 \mathrm{GeV}$, the 1999-2000 period $4<Q^{2}<80 \mathrm{GeV}^{2}$ and $30<W<$ $140 \mathrm{GeV}$; in both cases $|t|<1 \mathrm{GeV}^{2}$.

The $\gamma^{*} p$ cross section is shown differentially in $t$ in Fig. 4 and given in Table 1 for $Q^{2}=4 \mathrm{GeV}^{2}$ and $W=$ $71 \mathrm{GeV}$ (using the 1996-1997 data) and $Q^{2}=8 \mathrm{GeV}^{2}$ and $W=82 \mathrm{GeV}$ (using the 1999-2000 data). The data points are fitted with the exponential form $e^{-b|t|}$, which gives $b=6.66 \pm 0.54 \pm 0.43 \mathrm{GeV}^{-2}$ at $Q^{2}=4 \mathrm{GeV}^{2}$ where the first error is statistical and the second systematic. At $Q^{2}=$ $8 \mathrm{GeV}^{2}$, a value of $b=5.82 \pm 0.59 \pm 0.50 \mathrm{GeV}^{-2}$ is obtained. The two cross sections are averaged after correcting the 1996-1997 results to $Q^{2}=8 \mathrm{GeV}^{2}$ and $W=82 \mathrm{GeV}$ using equation (9) (see Table 1). The $t$ slope is then measured to be $b=6.02 \pm 0.35 \pm 0.39 \mathrm{GeV}^{-2}$.

The cross section as a function of $Q^{2}$ is shown in Fig. 5 and given in Table 2 for $W=82 \mathrm{GeV}$ and $|t|<1 \mathrm{GeV}^{2}$. Fitting the $Q^{2}$ dependence with the form $\left(1 / Q^{2}\right)^{n}$ gives $n=1.54 \pm 0.09 \pm 0.04$. The $Q^{2}$ dependence of the cross section is also given for a fixed value of $x_{B j}=1.8 \cdot 10^{-3}$ in Table 2 , in the restricted $Q^{2}$ range accessible for fixed $x_{B j}$.

The cross section as a function of $W$ is shown in Fig. 6 and given in Table 3 for $Q^{2}=4 \mathrm{GeV}^{2}$ and $Q^{2}=8 \mathrm{GeV}^{2}$; in both cases $|t|<1 \mathrm{GeV}^{2}$. The data are fitted using the form $W^{\delta}$ which gives $\delta=0.69 \pm 0.32 \pm 0.17$ at $Q^{2}=4 \mathrm{GeV}^{2}$

Table 1. Cross sections differential in $t$ for the two data samples and for the combined sample. The first errors are statistical, the second systematic

\begin{tabular}{|c|c|c|c|c|c|c|c|c|c|}
\hline & & & & $\gamma^{*} p \rightarrow$ & $\gamma p) / d t$ & {$[\mathrm{nb} / \mathrm{Ge}$} & & & \\
\hline & & $1996-19$ & & & 999-20 & & & All dat & \\
\hline & & $2=4 \mathrm{G}$ & & & $2=8 \mathrm{G}$ & & & $2=8 \mathrm{G}$ & \\
\hline$|t|\left[\mathrm{GeV}^{2}\right]$ & & $I=71 \mathrm{C}$ & & & $=82$ & & & $=82 C$ & \\
\hline $\begin{array}{l}0.1 \\
0.3\end{array}$ & $\begin{array}{l}29.9 \\
8.0\end{array}$ & $\begin{array}{l} \pm 4.1 \\
\pm 1.4\end{array}$ & $\begin{array}{l} \pm 7.1 \\
\pm 1.4\end{array}$ & $\begin{array}{l}13.3 \\
3.99\end{array}$ & $\begin{array}{l} \pm 1.9 \\
\pm 0.57\end{array}$ & $\begin{array}{l} \pm 3.4 \\
\pm 0.69\end{array}$ & $\begin{array}{l}12.0 \\
3.44\end{array}$ & $\begin{array}{l} \pm 1.2 \\
\pm 0.38\end{array}$ & $\begin{array}{l} \pm 2.9 \\
\pm 0.61\end{array}$ \\
\hline $\begin{array}{l}0.3 \\
0.5\end{array}$ & 2.13 & \pm 0.60 & \pm 0.69 & 0.90 & \pm 0.25 & \pm 0.30 & 0.84 & \pm 0.17 & \pm 0.29 \\
\hline 0.8 & 0.27 & \pm 0.12 & \pm 0.14 & 0.36 & \pm 0.09 & \pm 0.14 & 0.21 & \pm 0.04 & \pm 0.09 \\
\hline
\end{tabular}


Table 2. The $\gamma^{*} p \rightarrow \gamma p$ cross section as a function of $Q^{2}$ for $|t|<1 \mathrm{GeV}^{2}$, at $W=82 \mathrm{GeV}$ (second column) and at $x_{B j}=1.8 \cdot 10^{-3}$ (third column). The first errors are statistical, the second systematic

\begin{tabular}{|c|c|c|c|c|c|c|}
\hline \multirow{3}{*}{$\frac{Q^{2}\left[\mathrm{GeV}^{2}\right]}{3.0}$} & \multicolumn{6}{|c|}{$\sigma\left(\gamma^{*} p \rightarrow \gamma p\right) \quad[\mathrm{nb}]$} \\
\hline & \multicolumn{3}{|c|}{$W=82 \mathrm{GeV}$} & \multicolumn{3}{|c|}{$x_{B j}=1.8 \cdot 10^{-3}$} \\
\hline & 15.7 & \pm 2.5 & \pm 3.4 & & & \\
\hline 5.25 & 5.7 & \pm 1.1 & \pm 1.4 & 6.74 & \pm 0.93 & \pm 1.02 \\
\hline 8.75 & 3.20 & \pm 0.49 & \pm 0.69 & 3.25 & \pm 0.51 & \pm 0.60 \\
\hline 15.5 & 1.20 & \pm 0.22 & \pm 0.32 & 1.45 & \pm 0.30 & \pm 0.36 \\
\hline 25.0 & 0.70 & \pm 0.19 & \pm 0.19 & & & \\
\hline 55.0 & 0.15 & \pm 0.05 & \pm 0.05 & & & \\
\hline
\end{tabular}

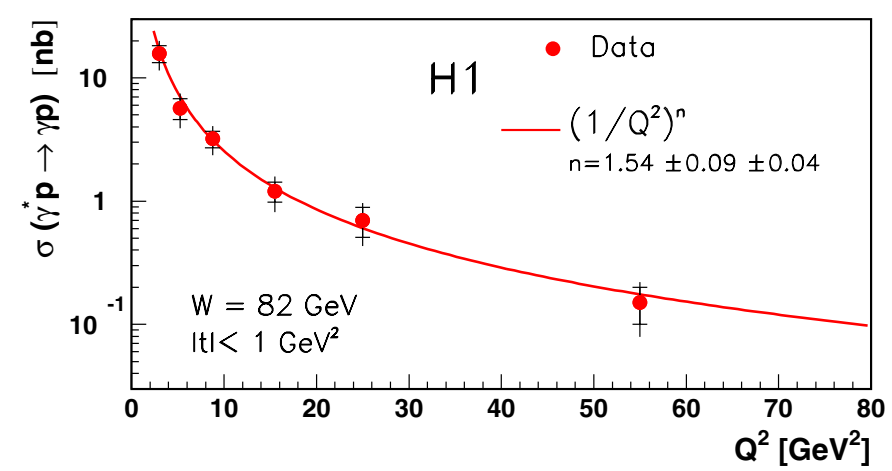

Fig. 5. The $\gamma^{*} p \rightarrow \gamma p$ cross section as a function of $Q^{2}$ for $W=82 \mathrm{GeV}$ and $|t|<1 \mathrm{GeV}^{2}$. The inner error bars represent the statistical and the full error bars the quadratic sum of the statistical and systematic uncertainties. The curve is the result of a fit to the form $\left(1 / Q^{2}\right)^{n}$, giving the value of $n$ shown in the figure (see Table 4)

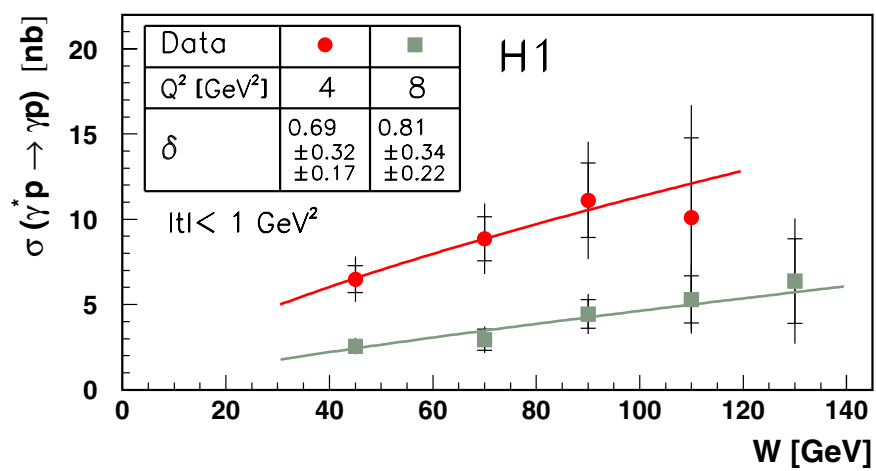

Fig. 6. The $\gamma^{*} p \rightarrow \gamma p$ cross section as a function of $W$ for $|t|<1 \mathrm{GeV}^{2}$ at $Q^{2}=4 \mathrm{GeV}^{2}$ and at $Q^{2}=8 \mathrm{GeV}^{2}$. The inner error bars represent the statistical and the full error bars the statistical and systematic uncertainties added in quadrature. The lines are the results of a fit to the form $W^{\delta}$, giving the values of $\delta$ shown in the insert (see Table 4 )

and $\delta=0.81 \pm 0.34 \pm 0.22$ at $Q^{2}=8 \mathrm{GeV}^{2}$. The two measurements are combined as explained above and the resulting cross section is given in Table 3 at $Q^{2}=8 \mathrm{GeV}^{2}$. Fitting the combined sample with the form $W^{\delta}$ gives $\delta=$ $0.77 \pm 0.23 \pm 0.19$. The steep rise of the cross section with $W$ is a strong indication of the presence of a hard scattering process, the value of $\delta$ being comparable to that measured in exclusive $J / \psi$ production $[46,47]$.

The extracted values of $b, \delta$ and $n$ are summarised in Table 4.

Table 3. The $\gamma^{*} p \rightarrow \gamma p$ cross section as a function of $W$ for $|t|<1 \mathrm{GeV}^{2}$ for the two data samples and for the combined sample. The first errors are statistical, the second systematic

\begin{tabular}{|c|c|c|c|c|c|c|c|c|c|}
\hline \multirow{4}{*}{$\frac{}{W[\mathrm{GeV}]}$} & \multicolumn{9}{|c|}{$\sigma\left(\gamma^{*} p \rightarrow \gamma p\right) \quad[\mathrm{nb}]$} \\
\hline & \multicolumn{3}{|c|}{ 1996-1997 } & \multicolumn{3}{|c|}{ 1999-2000 } & \multicolumn{3}{|c|}{ All data } \\
\hline & \multicolumn{3}{|c|}{$Q^{2}=4 \mathrm{GeV}^{2}$} & \multicolumn{3}{|c|}{$Q^{2}=8 \mathrm{GeV}^{2}$} & \multicolumn{3}{|c|}{$Q^{2}=8 \mathrm{GeV}^{2}$} \\
\hline & 6.5 & \pm 0.8 & \pm 1.1 & 2.56 & \pm 0.36 & \pm 0.32 & 2.28 & \pm 0.21 & \pm 0.34 \\
\hline 70 & 8.9 & \pm 1.3 & \pm 1.6 & 2.93 & \pm 0.63 & \pm 0.46 & 2.91 & \pm 0.35 & \pm 0.51 \\
\hline 90 & 11.1 & \pm 2.2 & \pm 2.7 & 4.45 & \pm 0.83 & \pm 0.82 & 3.97 & \pm 0.54 & \pm 0.85 \\
\hline 110 & 10.1 & \pm 4.7 & \pm 4.6 & 5.3 & \pm 1.4 & \pm 1.4 & 4.4 & \pm 1.0 & \pm 1.5 \\
\hline 130 & & & & 6.4 & \pm 2.5 & \pm 2.7 & 6.4 & \pm 2.5 & \pm 2.7 \\
\hline
\end{tabular}

Table 4. Summary of the $b, \delta$ and $n$ values separately for the two data taking periods at $Q^{2}=4 \mathrm{GeV}^{2}$ and $Q^{2}=8 \mathrm{GeV}^{2}$ and for the combined sample at $Q^{2}=8 \mathrm{GeV}^{2}$. The first errors are statistical, the second systematic. The values of $b$ are measured at $W=71 \mathrm{GeV}$ for $Q^{2}=4$ and $W=82 \mathrm{GeV}$ for $Q^{2}=8$. The values of $\delta$ and $n$ are given for $|t|<1 \mathrm{GeV}^{2}$. The value of $n$ is calculated at $W=82 \mathrm{GeV}$

\begin{tabular}{llll}
\hline$Q^{2}$ & $b\left[\mathrm{GeV}^{-2}\right]$ & $\delta$ & $n$ \\
\hline $4 \mathrm{GeV}^{2}$ & $6.66 \pm 0.54 \pm 0.43$ & $0.69 \pm 0.32 \pm 0.17$ & \\
$8 \mathrm{GeV}^{2}$ & $5.82 \pm 0.59 \pm 0.50$ & $0.81 \pm 0.34 \pm 0.22$ & $1.54 \pm 0.09 \pm 0.04$ \\
All data, $8 \mathrm{GeV}^{2}$ & $6.02 \pm 0.35 \pm 0.39$ & $0.77 \pm 0.23 \pm 0.19$ & \\
\hline
\end{tabular}



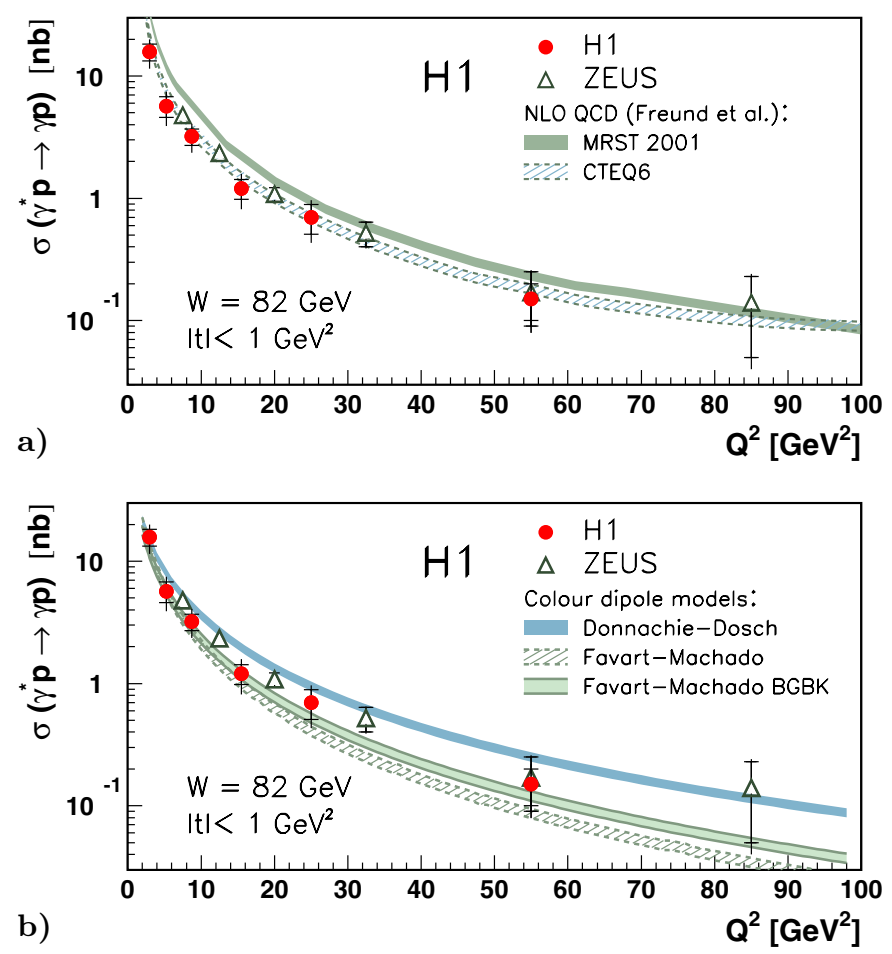

Fig. 7. The $\gamma^{*} p \rightarrow \gamma p$ cross section as a function of $Q^{2}$ for $W=82 \mathrm{GeV}$ and $|t|<1 \mathrm{GeV}^{2}$. The inner error bars represent the statistical and the full error bars the statistical and systematic uncertainties added in quadrature. The $\mathrm{H} 1$ measurement is shown together with the results of ZEUS [12] and several theoretical predictions. a Comparison with QCD predictions calculated at NLO by Freund et al. [25] based on MRST 2001 and CTEQ6 PDFs. b Comparison with the colour dipole predictions of Donnachie and Dosch [29] and Favart and Machado with [32] and without [30] the DGLAP evolution of the saturating dipole (indicated as BGBK). The band associated with each prediction corresponds to the uncertainty on the measured $t$-slope

\subsection{Discussion}

The cross section measurements from the combined data sample are shown with ZEUS measurements ${ }^{8}[12]$ and theoretical predictions as a function of $Q^{2}$ in Fig. 7a and as a function of $W$ in Fig. 8a. All predictions are made assuming an exponential dependence on $|t|$, using the measured value $b=6.02 \pm 0.52 \mathrm{GeV}^{-2}$. The error represents the total uncertainty of the $t$ slope which is reflected in the band associated with each of the predicted curves. The H1 and ZEUS measurements are seen to be consistent. The NLO QCD calculations of Freund et al. use two different GPDs, based on MRST 2001 and CTEQ6, for the diagonal distributions in the DGLAP domain. These two parameterisations show similar behaviour in $Q^{2}$ and in $W$ and differ mainly in the normalisation, which reflects the relative size of the quark singlet and gluon distributions for

\footnotetext{
8 The ZEUS measurements, for $W=89 \mathrm{GeV}$, have been rescaled to $W=82 \mathrm{GeV}$ and from $Q^{2}=9.6 \mathrm{GeV}^{2}$ to $Q^{2}=$ $8 \mathrm{GeV}^{2}$ using the parameter values $\delta=0.75$ and $n=1.54$ as quoted by ZEUS.
}
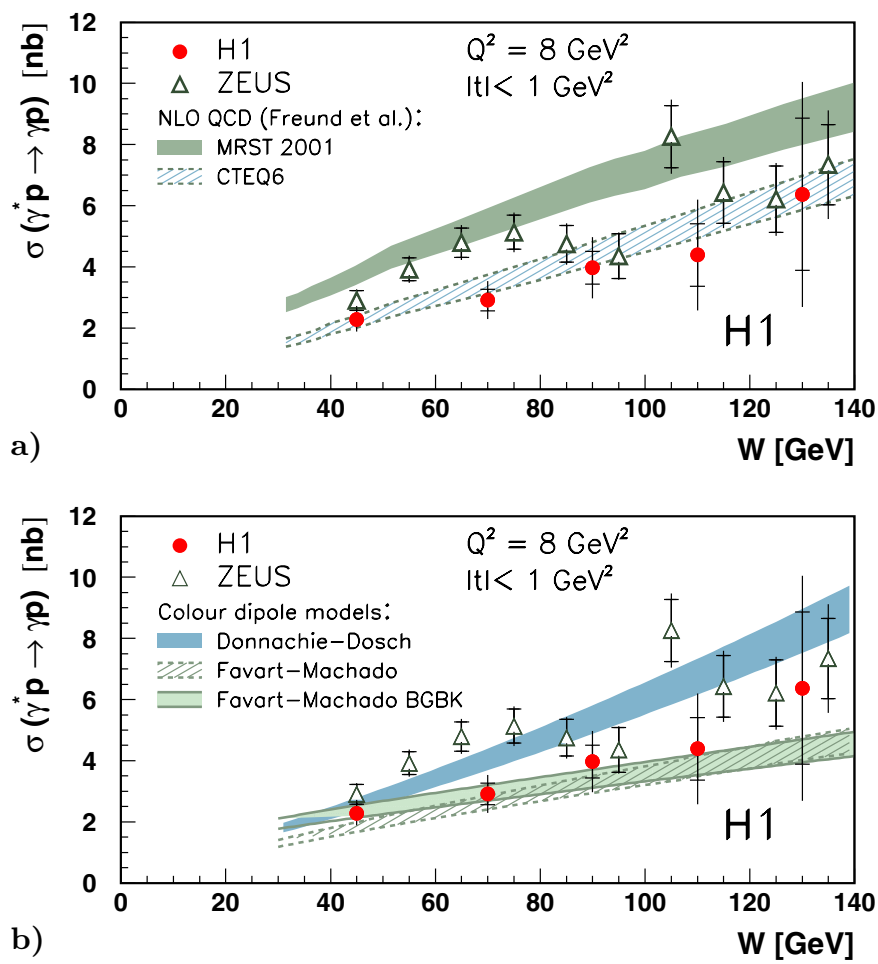

Fig. 8. The $\gamma^{*} p \rightarrow \gamma p$ cross section as a function of $W$ for $Q^{2}=8 \mathrm{GeV}^{2}$ and $|t|<1 \mathrm{GeV}^{2}$. The inner error bars represent the statistical and the full error bars the statistical and systematic uncertainties added in quadrature. The measurement is shown with the results of ZEUS [12] and several theoretical predictions. a Comparison with QCD predictions calculated at NLO by Freund et al. [25] based on MRST 2001 and CTEQ6 PDFs. $\mathbf{b}$ Comparison with the colour dipole predictions of Donnachie and Dosch [29] and Favart and Machado with [32] and without [30] the DGLAP evolution of the saturating dipole (indicated as BGBK). The band associated with each prediction corresponds to the uncertainty on the measured $t$-slope

each set. The H1 data are better described by the parameterisation based on CTEQ6, but it must be noted that the prediction also depends on the parameterisation of the ERBL region. It is interesting to note that the NLO QCD prediction describes the data down to the lowest $Q^{2}$ values, i.e. $3 \mathrm{GeV}^{2}$. This indicates that, within the present experimental uncertainties, a fully perturbative approach is successful and that non perturbative corrections are not needed to describe the data.

As shown in Figs. 7b and 8b, colour dipole models also provide a reasonable description of the data, both in shape and in normalisation. The $Q^{2}$ dependence is better described by the Favart-Machado prediction when DGLAP evolution of the dipole (BGBK) is included. As regards the $W$ dependence, the $\mathrm{H} 1$ data are consistent with both the Donnachie-Dosch and the Favart-Machado predictions, while the ZEUS measurements slightly favour the Donnachie-Dosch prediction.

Introducing a $Q^{2}$ dependence of the $|t|$ slope,$b=$ $b_{0}\left(1-0.15 \log \left(Q^{2} / 2\right)\right) \mathrm{GeV}^{-2}[24]$, as extracted for exclusive $\rho$ meson production $[48,49]$ (with $b_{0}$ such that 
$b=6.02 \mathrm{GeV}^{-2}$ at $Q^{2}=8 \mathrm{GeV}^{2}$ ), does not significantly change the above conclusions.

\section{Conclusion}

The DVCS process has been studied in the kinematic region $30<W<140 \mathrm{GeV}, 2<Q^{2}<80 \mathrm{GeV}^{2}$ and $|t|<1 \mathrm{GeV}^{2}$ using data taken with the $\mathrm{H} 1$ detector in the years 1996 to 2000. The $\gamma^{*} p \rightarrow \gamma p$ cross section has been measured as a function of $Q^{2}$ and as a function of $W$, and for the first time differentially in $t$. The dependence of the cross section on $Q^{2}$ is well reproduced by the shape $\left(1 / Q^{2}\right)^{n}$ with $n=1.54 \pm 0.09 \pm 0.04$ at $W=82 \mathrm{GeV}$. The $W$ dependence can be described by a fit of the form $W^{\delta}$ yielding $\delta=0.77 \pm 0.23 \pm 0.19$ at $Q^{2}=8 \mathrm{GeV}^{2}$. The fall of the cross section differential in $t$ can be described by the form $e^{-b|t|}$ with $b=6.02 \pm 0.35 \pm 0.39 \mathrm{GeV}^{-2}$ at $Q^{2}=8 \mathrm{GeV}^{2}$. This first measurement of the $t$ dependence of DVCS constrains the normalisation of the theoretical predictions. NLO QCD calculations give a good description of the normalisation as well as of the $Q^{2}$ and $W$ dependence of the measured cross section using a parameterisation of the GPDs based on the CTEQ6 parton distribution functions. The calculations rely on ordinary (unskewed) parton distributions in the DGLAP region and generate the skewedness dynamically. Colour dipole model predictions also give a good general description of the data. This is particularly true for a saturation model in which the DGLAP equation is used to describe the evolution of the dipole.

Acknowledgements. We are grateful to the HERA machine group whose outstanding efforts have made and continue to make this experiment possible. We thank the engineers and technicians for their work in constructing and now maintaining the $\mathrm{H} 1$ detector, our funding agencies for financial support, the DESY technical staff for continual assistance and the DESY directorate for the hospitality which they extend to the non-DESY members of the collaboration. We are grateful to M. Diehl and A. Freund for valuable discussions. We thank A. Freund and M. McDermott for providing the NLO QCD predictions used in this analysis.

\section{References}

1. D. Müller et al., Fortsch. Phys. 42, 101 (1994) [hep$\mathrm{ph} / 9812448]$

2. X. Ji, Phys. Rev. Lett. 78, 610 (1997) [hep-ph/9603249]

3. A.V. Radyushkin, Phys. Rev. D 56, 5524 (1997) [hep$\mathrm{ph} / 9704207]$

4. For a review see e.g. M. Diehl, Phys. Rep. 388, 41 (2003), DESY-THESIS-2003-018 [hep-ph/0307382].

5. X. Ji, Phys. Rev. D 55, 7114 (1997) [hep-ph/9609381]

6. J.C. Collins, A. Freund, Phys. Rev. D 59, 074009 (1999) [hep-ph/9801262]

7. X. Ji, J. Osborne, Phys. Rev. D 58, 094018 (1998) [hep$\mathrm{ph} / 9801260]$

8. J. Blümlein, D. Robaschik, Nucl. Phys. B 581, 449 (2000) [hep-ph/0002071]
9. L. Mankiewicz et al., Phys. Lett. B 425, 186 (1998) [hep$\mathrm{ph} / 9712251]$

10. A.V. Belitsky, D. Müller, L. Niedermeier, A. Schäfer, Phys. Lett. B 474, 163 (2000) [hep-ph/9908337].

11. C. Adloff et al. [H1 Collaboration], Phys. Lett. B 517, 47 (2001) [hep-ex/0107005]

12. S. Chekanov et al. [ZEUS collaboration], Phys. Lett. B 573, 46 (2003) [hep-ex/0305028]

13. A. Airapetian et al. [HERMES Collaboration], Phys. Rev. Lett. 87, 182001 (2001) [hep-ex/0106068]

14. S. Stepanyan et al. [CLAS Collaboration], Phys. Rev. Lett. 87, 182002 (2001) [hep-ex/0107043].

15. X.D. Ji, J. Phys. G 24, 1181 (1998) [hep-ph/9807358]

16. V.N. Gribov, L.N. Lipatov, Yad. Fiz. 15, 781 (1972) [Sov. J. Nucl. Phys. 15, 438 (1972)]

17. L.N. Lipatov, Yad. Fiz. 20, 181 (1974) [Sov. J. Nucl. Phys. 20, 94 (1975)]

18. G. Altarelli, G. Parisi, Nucl. Phys. B 126, 298 (1977)

19. Y.L. Dokshitzer, Zh. Eksp. Teor. Fiz. 73, 1216 (1977) [Sov. Phys. JETP 46, 641 (1977)]

20. A.V. Efremov, A.V. Radyushkin, Phys. Lett. B 94, 245 (1980)

21. G.P. Lepage, S.J. Brodsky, Phys. Lett. B 87, 359 (1979).

22. X. Ji, Phys. Rev. Lett. 78, 610 (1997) [hep-ph/9603249]; P. Hoodbhoy, X. Ji, W. Lu, Phys. Rev. D 59, 014013 (1999) and 074010 [hep-ph/9808305].

23. A. Freund, M.F. McDermott, Phys. Rev. D 65 (2002) 091901 [hep-ph/0106124]

24. A. Freund, M.F. McDermott, Phys. J. C 23 (2002) 651 [hep-ph/0111472]

25. A. Freund, M.F. McDermott, M. Strikman, Phys. Rev. D 67 (2003) 036001 [hep-ph/0208160]

26. A.D. Martin, R.G. Roberts, W.J. Stirling, R.S. Thorne, Eur. Phys. J. C 28, 455 (2003) [hep-ph/0211080]

27. D. Stump, J. Huston, J. Pumplin, W.-K. Tung, H.L. Lai, S. Kuhlmann, J.F. Owens, JHEP 0310, 046 (2003) [hep$\mathrm{ph} / 0303013]$

28. A.V. Belitsky, D. Müller, A. Kirchner, Nucl. Phys. B 629, 323 (2002) [hep-ph/0112108].

29. A. Donnachie, H.G. Dosch, Phys. Lett. B 502, 74-78 (2001) [hep-ph/0010227]

30. L. Favart, M.V. Machado, Eur. Phys. J. C 29, 365-371 (2003) [hep-ph/0302079]

31. K. Golec-Biernat, M. Wüsthoff, Phys. Rev. D 60, 114023 (1999) [hep-ph/9903358]

32. L. Favart, M.V. Machado, Eur. Phys. J. C 34, 429 (2004) [hep-ph/0402018]

33. J. Bartels, K. Golec-Biernat, H. Kowalski, Acta Phys. Polon. B 33, 2853 (2002) [hep-ph/0207031].

34. I. Abt et al. [H1 Collaboration], Nucl. Instrum. Meth. A 386, 310 (1997) and 348.

35. R.D. Appuhn et al. [H1 SPACAL Group], Nucl. Instrum. Meth. A 386, 397 (1997)

36. B. Andrieu et al. [H1 Calorimeter Group], Nucl. Instrum. Meth. A 350, 57 (1994).

37. E. Perez, L. Schoeffel, L. Favart, DESY-04-228 [hep$\mathrm{ph} / 0411389]$.

38. A. Freund, Phys. Rev. D 68, 096006 (2003) [hep$\mathrm{ph} / 0306012]$.

39. X. Janssen, Ph.D. Thesis, Université Libre de Bruxelles, 2003, DESY-THESIS-2003-004, available through: http://www-h1.desy.de/publications/theses_list.html 
40. A. Courau, S. Kermiche, T. Carli, P. Kessler, Quasi-Real QED Compton Monte Carlo, Proceedings of the Workshop on Physics at HERA, eds. W. Buchmüller, G. Ingelman, Vol. 2: p. 902, Hamburg 1991

41. V. Lendermann, Ph.D. Thesis, Universität Dortmund, 2002, DESY-THESIS-2002-004, available through: http://www-h1.desy.de/publications/theses_list.html

42. A. Mücke et al., Comput. Phys. Commun. 124 (2000) 290 [astro-ph/9903478]

43. B. List, A. Mastroberardino, DIFFVM: A Monte Carlo generator for diffractive processes in ep scattering, Proceedings of the DESY workshop on Monte Carlo Generators for HERA Physics, eds. A. T. Doyle, G. Grindhammer, G. Ingelman, H. Jung, DESY-PROC-1999-02, p. 396
44. R. Stamen, Ph.D. Thesis, Universität Dortmund, DESYTHESIS-2001-057, available through: http://wwwh1.desy.de/publications/theses_list.html

45. L.N. Hand, Phys. Rev. 129, 1834 (1963).

46. C. Adloff et al. [H1 Collaboration], Eur. Phys. J. C 10, 373 (1999) [hep-ex/9903008]

47. S. Chekanov et al. [ZEUS Collaboration], Eur. Phys. J. C 24, 345 (2002) [hep-ex/0201043]

48. C. Adloff et al. [H1 Collaboration], Eur. Phys. J. C 13, 371 (2000) [hep-ex/9902019]

49. J. Breitweg et al. [ZEUS Collaboration], Eur. Phys. J. C 6, 603 (1999) [hep-ex/9808020] 